\title{
CORRECTION
}

\section{Correction to: Hyperbolicity of the partition Jensen polynomials}

Hannah Larson and lan Wagner*

${ }^{*}$ Correspondence: iwagner@emory.edu

Emory University, Atlanta, USA

\section{Correction to: Larson, Wagner Res. Number Theory (2019) 5:19 https://doi.org/10.1007/s40993-019-0157-y}

There is a minor typographical error on page 3 which says that the hyperbolicity of the polynomial $P(X)$ is equivalent to $D_{d, m}(P(X)) \geq 0$ for all $m \leq d$. The correct statement is that the hyperbolicity of $P(X)$ is implied by all $D_{d, m}(P(X))$ being strictly positive. This does not change any of the main results of the paper. The authors would like to thank Jacques Gélinas for pointing out this mistake.

The original article can be found online at https://doi.org/10.1007/s40993-019-0157-y.

Published online: 17 June 2019

Publisher's Note

Springer Nature remains neutral with regard to jurisdictional claims in published maps and institutional affiliations. 